\title{
Unusual Features of QCD Low-Energy Modes in the Infrared Phase
}

\author{
Andrei Alexandru ${ }^{1, *}$ and Ivan Horváth ${ }^{2, \dagger}$ \\ ${ }^{1}$ The George Washington University, Washington, D.C. 20052, USA \\ ${ }^{2}$ University of Kentucky, Lexington, Kentucky 40506, USA
}

(Received 25 March 2021; accepted 28 June 2021; published 28 July 2021; corrected 3 August 2021)

\begin{abstract}
It was recently proposed that there is a phase in thermal QCD (IR phase) at temperatures well above the chiral crossover, featuring elements of scale invariance in the infrared (IR). Here, we study the effective spatial dimensions $d_{\mathrm{IR}}$ of Dirac low-energy modes in this phase, in the context of pure-glue QCD. Our $d_{\mathrm{IR}}$ is based on the scaling of mode support toward thermodynamic limit, and hence is an IR probe. Ordinary extended modes, such as those at high energy, have $d_{\mathrm{IR}}=3$. We find $d_{\mathrm{IR}}<3$ in the spectral range whose lower edge coincides with $\lambda_{\mathrm{IR}}=0$, the singularity of spectral density defining the IR phase, and the upper edge with $\lambda_{A}$, the previously identified Anderson-like nonanalyticity. Details near $\lambda_{\mathrm{IR}}$ are unexpected in that only exact zero modes are $d_{\mathrm{IR}}=3$, while a thin spectral layer near zero is $d_{\mathrm{IR}}=2$, followed by an extended layer of $d_{\mathrm{IR}}=1$ modes. With only integer values appearing, $d_{\mathrm{IR}}$ may have a topological origin. We find similar structure at $\lambda_{A}$, and associate its adjacent thin layer $\left(d_{\mathrm{IR}} \gtrsim 2\right)$ with Anderson-like criticality. Our analysis reveals the manner in which nonanalyticities at $\lambda_{\mathrm{IR}}$ and $\lambda_{A}$, originally identified in other quantities, appear in $d_{\mathrm{IR}}(\lambda)$. This dimension structure may be important for understanding the near-perfect fluidity of the quark-gluon medium seen in accelerator experiments. The role of $\lambda_{A}$ in previously conjectured decoupling of IR component is explained.
\end{abstract}

DOI: 10.1103/PhysRevLett.127.052303

Introduction.-The interest in Dirac eigenmodes of Euclidean quantum chromodynamics (QCD) has a long history, sparked in part by the role of zero modes in topology of gauge fields $\left(\eta^{\prime}\right.$ problem [1]) and by that of near-zero modes in spontaneous chiral symmetry breaking (Banks-Casher relation [2]). While modeling of low-energy QCD based on instantons could qualitatively accommodate these features [3], the birth of numerical lattice QCD [4] allowed for computation of Dirac eigenmodes from first principles [5,6]. This provided access to details of their true structure and thus an important window into the inner workings of QCD dynamics (see, e.g., [7-12]).

Recently, Dirac eigenmodes were used to infer the existence of a new phase in thermal QCD (IR phase) [13], showing certain signs of scale invariance at energies below temperature $T$. It was proposed that, past a crossover region (chiral $T_{c} \approx 155 \mathrm{MeV}$ ), a true QCD phase transition may occur at temperature $T_{\mathrm{IR}}(200-250 \mathrm{MeV})$, marking the restoration of scale invariance in the infrared (IR). The reasoning was based on the proposition that the observed power law behavior of Dirac spectral density in $\operatorname{IR}[\rho(\lambda) \approx 1 / \lambda]$ arises due to the underlying IR scale

Published by the American Physical Society under the terms of the Creative Commons Attribution 4.0 International license. Further distribution of this work must maintain attribution to the author(s) and the published article's title, journal citation, and DOI. Funded by SCOAP ${ }^{3}$. invariance of glue fields. This is corroborated by the finding that such a Dirac spectral feature also occurs near SU(3) conformal window at zero temperature $[11,14]$, placing both corners of the theory parameter space into one contiguous dynamical regime, the IR phase.

Given the relevance of the above to the physics of quarkgluon plasma studied at RHIC and LHC, as well as to the physics of the early Universe (see, e.g., [15] for review), our aim in this work is to describe the IR phase in a manner that sheds more light on the mechanism of its conjectured scale invariance. Owing to their proposed common origin, the new insight would also be valuable for understanding the mechanism of scale invariance in the strongly coupled part of the conformal window.

To work with a concrete problem, consider pure-glue QCD at $T>T_{\mathrm{IR}}$. Reference [13] proposes the existence of a physical energy scale $\Lambda_{\mathrm{IR}}=\Lambda_{\mathrm{IR}}(T) \lesssim T$, such that the theory is scale invariant at energies $E \lesssim \Lambda_{\mathrm{IR}}$. In this scenario, gauge coupling stops running below $\Lambda_{\mathrm{IR}}$, leading to nonanalyticity at this point. How could such a feature arise in QCD? Motivated by clean bimodality of $\rho(\lambda)$, Ref. [13] suggested that IR gauge fields decouple and fluctuate independently of the bulk in the IR phase. The ensuing mismatch between the two independent components of the system can then produce such nonanalyticity.

In what follows we make this proposal more precise and concrete by focusing on nonanalyticities of Dirac spectra. After all, a true nonanalyticity of running coupling would be reflected in all dynamical elements of the theory, 
especially in a Dirac eigensystem where the singularity of $\rho(\lambda)$ at $\lambda_{\mathrm{IR}}=0$ first suggested the existence of IR phase. Hence, we aim to identify these nonanalyticities and to determine their role in the above dynamical scenario.

In addition to $\lambda_{\mathrm{IR}}=0(\rho \approx 1 / \lambda)$, there is a well-known singularity of $\rho(\lambda)$ at $\lambda_{\mathrm{UV}}=\infty\left(\rho \approx \lambda^{3}\right)$. Moreover, a different type of spectral nonanalyticity, namely the Anderson-like localization point $\lambda_{A}>T$, has been advocated for and studied for some time $[10,16,17]$. While the singularity at $\lambda_{\mathrm{UV}}$ is expected at all temperatures (asymptotic freedom), $\lambda_{A}$ appears to be a companion of IR phase. If so, then $\lambda_{\mathrm{IR}}$ and $\lambda_{A}$ should be associated in some manner.

To identify such possible connection, as well as to search for additional nonanalyticities of Dirac spectra, we study the dimension $d_{\mathrm{IR}}=d_{\mathrm{IR}}(\lambda)$ of spatial region effectively occupied by modes. We emphasize that we use the infrared concept of dimension which probes the response of effective volume to the release of IR cutoff. This approach has its roots in Ref. [18] and will be discussed in Sec. 2. (The full account of dimension theory will be given in Ref. [19].) In Sec. 3 we describe our numerical results. Their proposed implications are elaborated upon in Sec. 4.

This work is concerned with results in pure-glue QCD. However, we believe that our findings carry over in the presence of dynamical quarks. This is only a conjecture at this stage, albeit a reasonable one given the likely topological nature of the reported features. We plan to investigate this issue in future studies.

Dimensions.-Consider thermal QCD in four-volume $L^{3} / T$, regularized on a hypercubic lattice with spacing $a$. For questions of thermodynamic $(L \rightarrow \infty)$ limit, scale $1 / L$ is the IR cutoff, similarly to $1 / a$ being the UV cutoff. The eigenmodes $D \psi_{\lambda}(x)=i \lambda \psi_{\lambda}(x)$ of a continuum Dirac operator $D$ have suitable lattice counterparts, supplied here by those of the overlap operator [20]. This ensures continuumlike chiral and topological properties.

Following [18,19], we assign spatial dimensions to Dirac eigenmodes as follows. Consider the eigenmode $\psi_{\lambda}=$ $\psi_{\lambda}\left(x_{i}, L, a\right)$ at given $\lambda$ and cutoffs. Although nominally such a mode extends over all $N=(L / a)^{3} /(T a)$ sites, this "counting" should be modified if the probabilities $P=\left(p_{1}, p_{2}, \ldots, p_{N}\right), p_{i}=\psi_{\lambda}^{+} \psi_{\lambda}\left(x_{i}\right)$, are effectively concentrated in fewer $(\mathcal{N}<N)$ sites. Assume that such effective counting $\mathcal{N}=\mathcal{N}[P]$ is in place. While $N \propto L^{3}$ at fixed $a$, the effective $\mathcal{N}$ is governed by $\mathcal{N} \propto L^{d_{\mathrm{IR}}}$ for $L \rightarrow \infty$, with $0 \leq d_{\mathrm{IR}} \leq 3$. Dimension $d_{\mathrm{IR}}=d_{\mathrm{IR}}(a)$ is "infrared" since it probes the removal of IR cutoff. Similarly, while $N \propto a^{-4}$, the power $d_{\mathrm{UV}}=d_{\mathrm{UV}}(L)$ in $\mathcal{N} \propto a^{-d_{\mathrm{UV}}}$ for $a \rightarrow 0\left(0 \leq d_{\mathrm{UV}} \leq 4\right)$ is the effective $\mathrm{UV}$ dimension.

Effective number theory [18] specifies all additive effective counting schemes $\mathcal{N}$, and leads to

$$
\mathcal{N}_{\star}[P]=\sum_{i=1}^{N} \mathfrak{n}_{\star}\left(N p_{i}\right), \quad \mathfrak{n}_{\star}(c)=\min \{c, 1\}
$$

for a scheme that consistently delimits the effective subset (support) of objects with probabilities [19]. This makes $d_{\mathrm{IR}}$, $d_{\mathrm{UV}}$ well-defined characteristics of the mode's effective support. Since $\mathcal{N}_{\star}$ is additive, $d_{\mathrm{UV}}$ and $d_{\mathrm{IR}}$ are stochastic measure-based constructs analogous to box-counting and Hausdorff dimensions [19]. (Reference [21] describes a similar approach based on the participation number. Because of the lack of additivity, it is not Hausdorff-like.) In fact, the method can be adapted to define dimensions of fixed (nonstochastic) fractal sets, and we verified in few cases that it produces $d_{\mathrm{UV}}$ consistent with their Hausdorff dimensions.

Here, we focus on IR dimensions of Dirac modes. Since $\psi_{\lambda}$ is a statistical object, $d_{\mathrm{IR}}$ is defined by

$$
\left\langle\mathcal{N}_{\star}\right\rangle_{a, L, \lambda} \propto L^{d_{\mathrm{IR}}(a, \lambda)} \text { for } L \rightarrow \infty,
$$

where $\langle\cdots\rangle_{a, L, \lambda}$ denotes the QCD average at fixed cutoffs, and the spectral average in the vicinity of $\lambda$.

The results.-We computed $d_{\mathrm{IR}}(\lambda)$ in the IR phase of pure-glue QCD $\left(T=1.12 T_{\mathrm{IR}}\right)$ using the setup of Ref. [13] (Wilson action at $\beta=6.054, a=0.085 \mathrm{fm}$ via $r_{0}=0.5 \mathrm{fm}$, overlap operator at $\left.\rho=26 / 19\right)$. Systems with $L / a=16,20,24,32,40,48,64$ and $1 /(T a)=7$ were analyzed. Special care was required to reliably identify the zero modes, and to implement the overlap operator in a numerically efficient way [22-24].

Our main results are conveyed by Fig. 1. Dimensions were obtained from fits to the asymptotic form (2) using four largest systems (range 2.7-5.4 fm) in the analysis. The fits had statistically acceptable $\chi^{2} /$ d.o.f. in general. The spectral intervals associated with plotted points are disjoint, and cover the region shown.

We find the spectral interval $\left(\lambda_{1}, \lambda_{2}\right)$ of low- $d_{\mathrm{IR}}$ modes $\left(d_{\mathrm{IR}}<3\right)$, featuring three regimes: the constant central plateau at $d_{\mathrm{IR}} \approx 1$ and two half-peaks on the sides ("left rise" and "right rise"). The details are as follows.

Zero modes: The overlap operator supports exact topological zero modes, separated from the rest of the spectrum

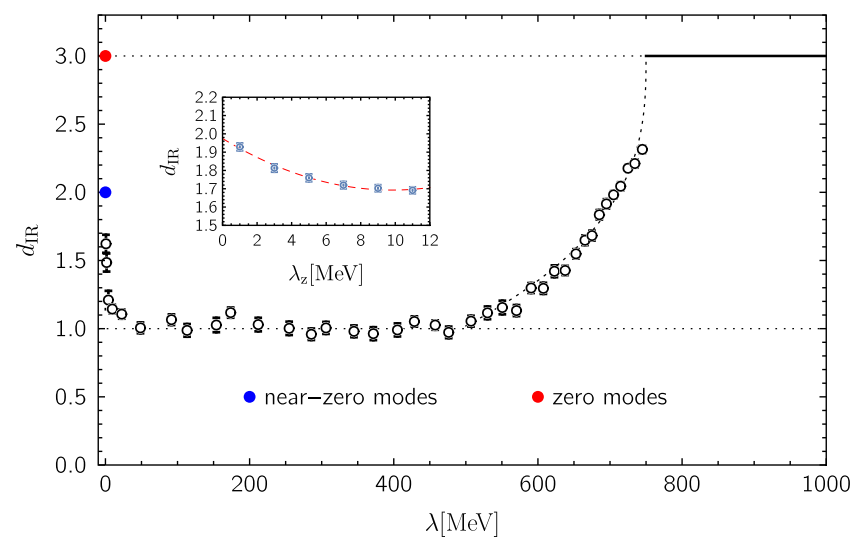

FIG. 1. IR dimension of Dirac modes at $T=1.12 T_{\mathrm{IR}}$. 


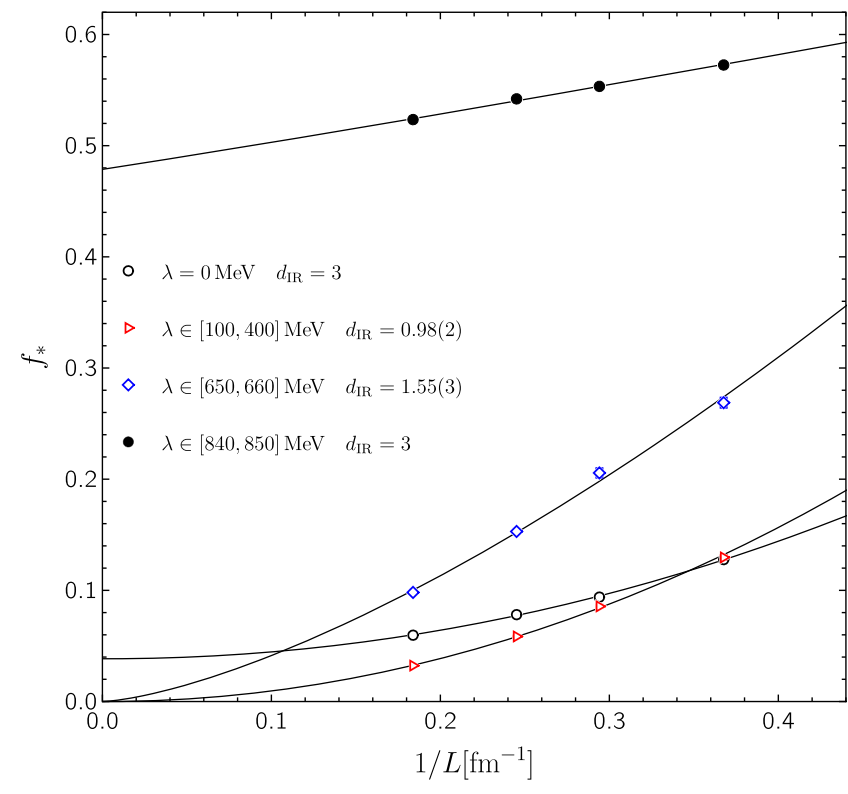

FIG. 2. The fraction $f_{\star}$ of space occupied by modes vs $1 / L$.

in any finite volume. We find that $d_{\mathrm{IR}}(0)=3$ (red point in Fig. 1), with the behavior of $f_{\star}=\mathcal{N}_{\star} / N \propto L^{d_{\mathrm{IR}}-3}$ shown in Fig. 2. Using the Anderson localization terminology, this means that zero modes are "extended" since they occupy a finite fraction of volume $(\approx 4 \%)$ in $L \rightarrow \infty$ limit.

Near-zero modes: Is there a layer of deep IR modes akin to strict zero modes in terms of $d_{\mathrm{IR}}$ ? To assess this, we treat the extent $\lambda_{\mathrm{z}}$ of such potential region $\left(0, \lambda_{\mathrm{z}}\right)$ as a parameter, and evaluate the average $d_{\mathrm{IR}}$ of modes from this interval. The result is shown in the inset of Fig. 1. It reveals that, rather than $d_{\mathrm{IR}}=3$, the deepest IR modes afforded by our data approach $d_{\mathrm{IR}}=2$, and hence $\lambda_{1}=0$. In the absence of evidence for its finite width, we represent the $d_{\mathrm{IR}}=2$ layer by a single (blue) point adjacent to point representing zero modes. Note that, although $d_{\mathrm{IR}}<3$ implies that quarks in these modes occupy a space of measure zero relative to the entire volume, they are not necessarily localized in terms of distances involved.

The left rise: Consider now the entire left rise in Fig. 1. Since its spectral extent ends just above $1 / L_{\max }$ $\left(L_{\max }=64 a\right)$, we need to ask whether the onset of the rise marks a nonzero IR scale or vanishes with IR cutoff. To assess this, we vary $L_{\max }$ by using sizes of smaller simulated systems as IR cutoffs in the dimension analysis. Fig. 3 (left) shows the dependence of $d_{\mathrm{IR}}$ on $L_{\max }$ for spectral range 5-15 MeV inside the rise. We observe lowering of the dimension toward $d_{\mathrm{IR}} \approx 1$ with increasing $L_{\max }$. The consistency of such trends makes us to suggest that the width of the left rise vanishes in thermodynamic limit and a new dynamical scale is not generated.

The upper edge: The low- $d_{\mathrm{IR}}$ regime in Fig. 1 ends abruptly at $\lambda_{2} \approx 750 \mathrm{MeV}$, suggesting a nonanalytic behavior. The position of the edge can be estimated by including a constant in fits of $f_{\star}$ (zeroth power in addition to floating

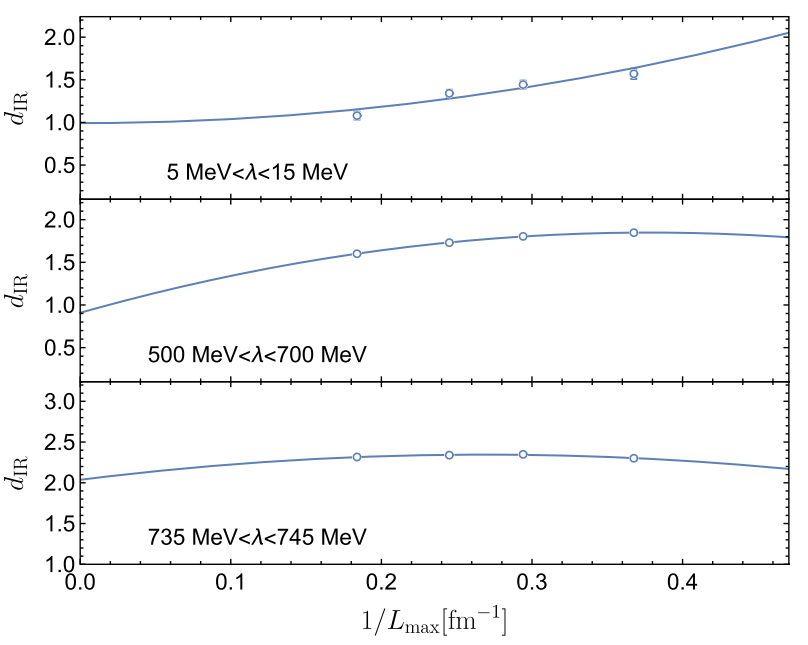

FIG. 3. $d_{\mathrm{IR}}$ as a function $1 / L_{\max }$ in left and right rises.

positive power). Figure 4 (top) shows this constant in the relevant spectral range. The small negative values below $\approx 750 \mathrm{MeV}$ indicate that the term is redundant since the leading contribution has to be positive on geometric grounds. Hence, $d_{\mathrm{IR}}<3$ in this region. A statistically significant positive value, on the other hand, implies $d_{\mathrm{IR}}=3$ and the extended modes. The approach to constant $f_{\star}>0$ in the extended regime (black horizontal line in Fig. 1) is exemplified in Fig. 2 (full circles).

The existence of Anderson-like point in QCD Dirac spectra, defined as $\lambda_{A}$ where the statistics of unfolded level spacings changes from Poisson $\left(\lambda<\lambda_{A}\right)$ to Dyson-Wigner $\left(\lambda>\lambda_{A}\right)$, has been concluded in Ref. [10]. To check whether the low- $d_{\mathrm{IR}}$ upper edge $\lambda_{2}$ can be identified with $\lambda_{A}$, we use their procedure and look for the transition in

$$
I_{s_{0}}(\lambda)=\int_{0}^{s_{0}} d s p(s, \lambda)
$$

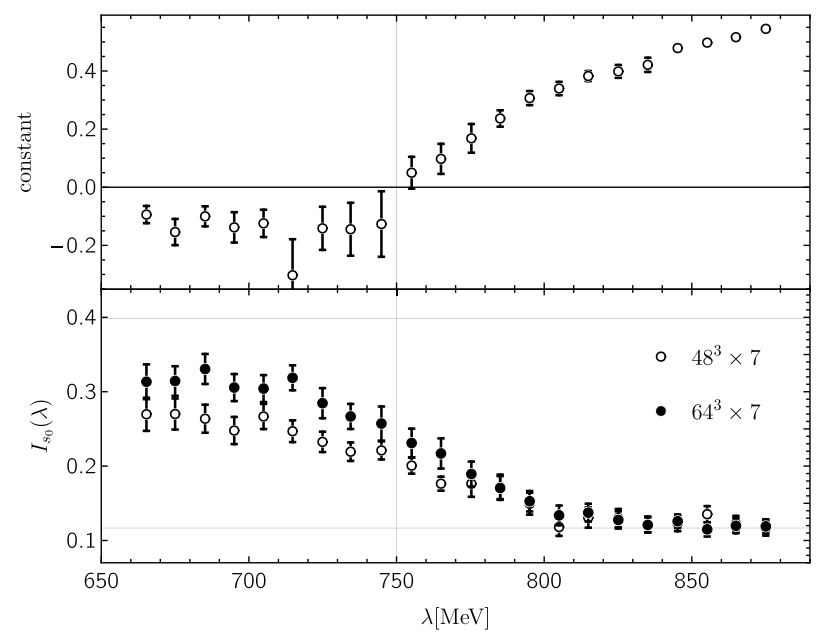

FIG. 4. The value of constant term in fits of $f_{\star}(1 / L)$ (top) and the value of $I_{0.508}$ (bottom) in the vicinity of $\lambda_{2}$. 
with $p(s, \lambda)$ the distribution of level spacings in the vicinity of $\lambda$. The value $s_{0} \approx 0.508$ maximizes the difference in $I_{s_{0}}$ for Poisson $\left(I_{0.508} \approx 0.398\right)$ and Dyson-Wigner unitary class $\left(I_{0.508} \approx 0.117\right)$ statistics. Figure 4 (bottom) shows $I_{0.508}(\lambda)$ for our two largest lattices. The approach to constant Dyson-Wigner value on the right is clear and the volume trend on the left is in the expected direction toward the Poisson value. The transition region is rather wide but approximately centered around $750 \mathrm{MeV}$. These results suggest that $\lambda_{2}$ and $\lambda_{A}$ represent the same spectral point in thermodynamic limit.

The right rise: The example of $f_{\star}(1 / L)$ inside the right rise is shown in Fig. 2. Like for the left rise, we need to inquire whether the spectral width of this feature remains finite in thermodynamic limit. Figure 3 (middle) shows $d_{\mathrm{IR}}$ versus floating $1 / L_{\max }$ for the spectral range $500-700 \mathrm{MeV}$ extending almost across the rise. A clear decrease of $d_{\mathrm{IR}}$ with increasing $L_{\max }$ is seen. The observed change is well described by quadratic polynomial, leading to $L_{\max } \rightarrow \infty$ value consistent with that of the plateau $\left(d_{\mathrm{IR}} \approx 1\right)$. On the other hand, very close to the edge (735-745) MeV, the same fitting suggests $d_{\mathrm{IR}} \gtrsim 2$. This leads us to propose that the left and right rises are similar in that the spectral widths of these features vanish in thermodynamic limit, each morphing into a pointlike layer of well-defined lower dimension.

The synthesis and discussion.-The analytic structure of the Dirac eigensystem can be useful for detecting the phases of QCD. For example, entering the IR phase by crossing $T_{\mathrm{IR}}$ is characterized by adding the IR power singularity of mode density $\rho(\lambda)$ to its already present $\mathrm{UV}$ singularity [13]. The $\lambda^{3}$ divergence at $\lambda_{\mathrm{UV}}=\infty$ reflects the strict power law $\rho(\lambda)=c \lambda^{3}$ directly at the Gaussian UV fixed point. Similarly, we associate the $1 / \lambda$ singularity at $\lambda_{\mathrm{IR}}=0$ with the power law at the strongly coupled IR fixed point governing the IR component of the system [13].

Here, we extended this analyticity angle by studying the spatial dimension $d_{\mathrm{IR}}(\lambda)$ of Dirac modes. The results lead us to propose that the IR phase of QCD is characterized by the existence of a spectral range $0 \leq \lambda_{1}<\lambda<\lambda_{2}$ of low- $d_{\text {IR }}$ modes. The edges $\lambda_{1}, \lambda_{2}$ are nonanalyticities of $d_{\mathrm{IR}}(\lambda)$. Our data are consistent with the following infinite-volume predictions. Prediction 1: $d_{\mathrm{IR}}=3$ for zero modes. Prediction 2: there is a pointlike layer of $d_{\mathrm{IR}}=2$ nearzero modes and hence $\lambda_{1}=\lambda_{\mathrm{IR}}=0$. We are not aware of any model that predicts this. Prediction 3: a plateau of $d_{\mathrm{IR}} \approx$ 1 modes spans the interior of low- $d_{\mathrm{IR}}$ interval. Prediction 4: $\lambda_{2}=\lambda_{A}$, where $\lambda_{A}$ is the Anderson-like transition point. Prediction 5: a pointlike layer of $d_{\mathrm{IR}} \gtrsim 2$ modes exists on the inner side of $\lambda_{2}$. The resulting $d_{\mathrm{IR}}(\lambda)$ is shown in Fig. 5 .

The study of $\rho(\lambda)$ and $d_{\mathrm{IR}}(\lambda)$ thus offers at least three Dirac nonanalyticities $\lambda_{\mathrm{IR}}=\lambda_{1}, \lambda_{A}=\lambda_{2}, \lambda_{\mathrm{UV}}$ in IR phase, with scales $T$ and $\Lambda_{\mathrm{IR}}(T)$ inside the low- $d_{\mathrm{IR}}$ range

$$
0=\lambda_{\mathrm{IR}}<\Lambda_{\mathrm{IR}}<T<\lambda_{A}<\lambda_{\mathrm{UV}}=\infty .
$$

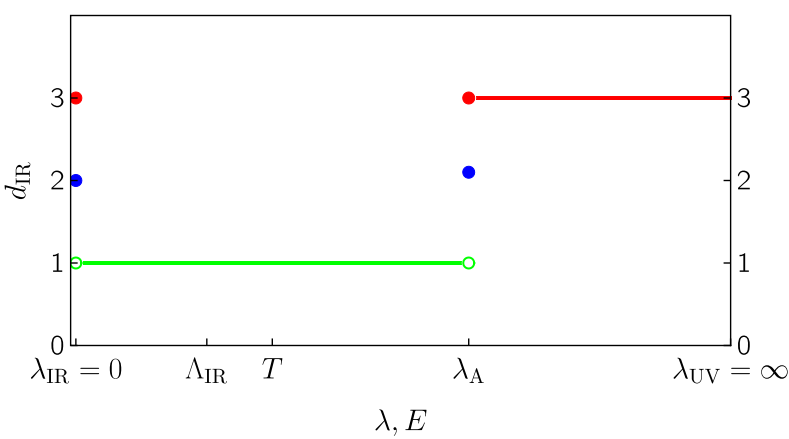

FIG. 5. Dimension $d_{\mathrm{IR}}$ of Dirac modes in the IR phase. The abscissa is common to physical energy scale $E$ and the Dirac $\lambda$.

While the presence of $\lambda_{\mathrm{IR}}$ as a singularity in $\rho(\lambda)$ is natural (see above), the purpose of $\lambda_{A}$ may seem puzzling. What is the role of a "phase transition" in the internal parameter $\lambda$ of the theory? Treating a QCD-induced Dirac dynamics near $\lambda_{A}$ as a model description of some system, the phase transition at $\lambda_{A}$ means that its "states" at $\lambda<\lambda_{A}$ are unrelated to those at $\lambda>\lambda_{A}$, which is further underlined here by the former being low $d_{\mathrm{IR}}$. We ascribe this mutual independence, realized by nonanalyticity at $\lambda_{A}$, to the decoupling of IR glue fields from the bulk. Hence, $\lambda_{A}$ is connected to the proposed mechanism of strongly coupled IR scale invariance [13], naturally tying with the nonanalyticity of running coupling at $\Lambda_{\mathrm{IR}}$.

We wish to make the following additional remarks. (a) The structure of $d_{\mathrm{IR}}(\lambda)$ is the same at $\lambda_{\mathrm{IR}}$ and $\lambda_{A}$, involving a two-step nonanalytic transition from nominal $d_{\mathrm{IR}}=3$ to low- $d_{\mathrm{IR}}$ regime. (b) The existence of the "middle point" at $\lambda_{A}$ is natural upon accepting its Anderson nature. Indeed, we associate it with the critical region in Anderson models [25], which shrink to zero width in thermodynamic limit. The observed $2 \lesssim d_{\mathrm{IR}}\left(\lambda_{A}\right)<3$ describes the system at strict criticality. (c) However, $d_{\mathrm{IR}} \approx 1$ below $\lambda_{A}$ is at odds with exponential localization in Anderson models. Clarifying this discrepancy is an interesting issue to resolve. (d) Despite $d_{\mathrm{IR}}$ being Hausdorff-like, only nearinteger dimensions appear around $\lambda_{\mathrm{IR}}$. Hence, IR dimension may have topological meaning for IR modes. The structure near $\lambda_{\mathrm{IR}}$ is interesting in that all "topological dimensions" occur. (e) The appearance of $d_{\mathrm{IR}}=2,1$ near $\lambda_{\mathrm{IR}}$ is unexpected in light of simple instanton-based models [3]. Adding this feature to model descriptions of IR component could be valuable since the latter may capture the near-perfect fluidity properties of the strongly interacting medium seen at accelerator experiments [13]. (f) The proposal for decoupling of IR component used the UV-IR bimodality of Dirac spectral density as its corroborating feature [13]. Since the position $0<\lambda_{\min }<T$ of minimal $\rho(\lambda)$ plays a special role here, it is desirable to clarify its status regarding analyticity. (g) Albeit indirectly and from different angles, recent works involving thermal Dirac modes [26-28] provide an additional information on IR 
phase. (h) We are not aware of a theoretical argument or lattice evidence at this time, suggesting that $d_{\mathrm{UV}}(\lambda, T)<4$ for any $\lambda, T$ in QCD.

A. A. is supported in part by the U.S. DOE Grant No. DE-FG02-95ER40907. I. H. acknowledges the discussions with Peter Markoš and Robert Mendris.

*aalexan@gwu.edu

ihorv2@g.uky.edu

[1] G. 't Hooft, Phys. Rev. Lett. 37, 8 (1976).

[2] T. Banks and A. Casher, Nucl. Phys. B169, 103 (1980).

[3] T. Schafer and E. V. Shuryak, Rev. Mod. Phys. 70, 323 (1998).

[4] M. Creutz, Phys. Rev. Lett. 43, 553 (1979); 43, 890(E) (1979).

[5] J. Smit and J. C. Vink, Nucl. Phys. B286, 485 (1987).

[6] J. Smit and J. Vink, Phys. Lett. B 194, 433 (1987).

[7] R. G. Edwards, U. M. Heller, J.E. Kiskis, and R. Narayanan, Phys. Rev. D 61, 074504 (2000).

[8] I. Horváth, S. J. Dong, T. Draper, N. Isgur, F. X. Lee, K. F. Liu, J. McCune, H. B. Thacker, and J. B. Zhang, Phys. Rev. D 66, 034501 (2002).

[9] A. Alexandru and I. Horváth, Phys. Lett. B 722, 160 (2013).

[10] M. Giordano, T. G. Kovacs, and F. Pittler, Phys. Rev. Lett. 112, 102002 (2014).

[11] A. Alexandru and I. Horváth, Nucl. Phys. B891, 1 (2015).

[12] A. Alexandru and I. Horváth, Phys. Rev. D 92, 045038 (2015).

[13] A. Alexandru and I. Horváth, Phys. Rev. D 100, 094507 (2019).

[14] A. Alexandru and I. Horváth, AIP Conf. Proc. 1701, 030008 (2016).
[15] W. Busza, K. Rajagopal, and W. van der Schee, Annu. Rev. Nucl. Part. Sci. 68, 339 (2018).

[16] A. M. Garcia-Garcia and J. C. Osborn, Phys. Rev. D 75, 034503 (2007).

[17] T. G. Kovacs and F. Pittler, Phys. Rev. Lett. 105, 192001 (2010).

[18] I. Horváth and R. Mendris, Entropy 22, 1273 (2020).

[19] I. Horváth and R. Mendris (to be published).

[20] H. Neuberger, Phys. Lett. B 417, 141 (1998).

[21] C. Aubin, C. Bernard, S. Gottlieb, E. B. Gregory, U. M. Heller, J. E. Hetrick, J. Osborn, R. Sugar, P. de Forcrand, and O. Jahn (MILC Collaboration), Nucl. Phys. B, Proc. Suppl. 140, 626 (2005).

[22] A. Alexandru, M. Lujan, C. Pelissier, B. Gamari, and F. X. Lee, in 2011 Symposium on Application Accelerators in High-Performance Computing (SAAHPC), Knoxville, TN, USA (IEEE, 2011), pp. 123-130.

[23] A. Alexandru, Comput. Sci. Eng. 17, 14 (2014).

[24] A. Alexandru, C. Pelissier, B. Gamari, and F. Lee, J. Comput. Phys. 231, 1866 (2012).

[25] F. Evers and A. D. Mirlin, Rev. Mod. Phys. 80, 1355 (2008).

[26] H. T. Ding, S. T. Li, S. Mukherjee, A. Tomiya, X. D. Wang, and Y. Zhang, Phys. Rev. Lett. 126, 082001 (2021).

[27] S. Aoki, Y. Aoki, G. Cossu, H. Fukaya, S. Hashimoto, T. Kaneko, C. Rohrhofer, and K. Suzuki (JLQCD Collaboration), Phys. Rev. D 103, 074506 (2021).

[28] R. A. Vig and T. G. Kovacs, Phys. Rev. D 103, 114510 (2021).

Correction: A misprint of a relation sign introduced during the production process in the first inline equation of the second sentence in the fourth paragraph has been fixed. 